Intradermal zoster vaccines: good for the old and the young?

Peer-reviewed author version

OGUNJIMI, Benson \& HENS, Niel (2016) Intradermal zoster vaccines: good for the old and the young?. In: LANCET INFECTIOUS DISEASES, 16(8), p. 869-871.

DOI: 10.1016/S1473-3099(16)00174-2

Handle: http://hdl.handle.net/1942/22709 


\title{
Intradermal zoster vaccines: good for the old and the young?
}

\author{
Benson Ogunjimi ${ }^{a}$, Niel Hens ${ }^{a, b}$ \\ ${ }^{a}$ University of Antwerp, Antwerpen, Belgium \\ ${ }^{\mathrm{b}}$ Center for Statistics, Hasselt University, Hasselt, Belgium \\ Corresponding author: niel.hens@uhasselt.be
}

In The Lancet Infectious Diseases, Chan R Beals and colleagues ${ }^{1}$ report the results of a clinical trial in which varicella-zoster virus specific immunogenicity and adverse events were compared between the Merck live attenuated full dose subcutaneous herpes zoster vaccine, $1 / 3$ subcutaneous zoster vaccine, full dose intradermal zoster vaccine, 1/3 intradermal zoster vaccine, 1/10 intradermal zoster vaccine, and 1/27 intradermal zoster vaccine. Full and 1/3 intradermal zoster vaccine caused a significantly higher increase in varicella-zoster virus antibody titer (measured by gpELISA) 6 weeks after vaccination compared with full subcutaneous zoster vaccine $(p<0.0001$ for full intradermal zoster vaccine and $p=0.007$ for the $1 / 3$ intradermal zoster vaccine). In a subgroup analysis, it was shown that after 18 months the gpELISA zoster vaccine titre was still higher after full and $1 / 3$ intradermal zoster vaccine compared with full subcutaneous varicella-zoster vaccine.

Varicella-zoster virus-specific interferon- $\gamma$ ELISPOT analyses detected no differences between the different vaccine formulations or administration routes 6 weeks after vaccination. Nevertheless, a flow cytometric analysis indicated that the proportion of varicella zoster virusspecific CD4+ central memory T-cells was significantly higher for the aggregated intradermal zoster vaccine results compared with the aggregated subcutaneous zoster vaccine results. Although both gpELISA varicella-zoster virus titres and varicella zoster virus-specific cellular mediated immunity have been shown to be associated with the risk of zoster, ${ }^{2}$ symptomatic varicella-zoster virus reactivation is mainly considered to be due to a decline in varicella zoster virus cellular mediated immunity.

In 2005, Oxman and colleagues ${ }^{3}$ presented the results of the administration of the subcutaneous zoster vaccine in a large cohort. Although the results showed a $61 \%$ reduction of zoster-related burden of illness in adults older than 60 years, follow-up studies showed an important waning of vaccine efficacy (zoster-related burden of illness zoster-vaccine efficacy of $37 \%$ in adults 60 years of age and older 11 years after vaccination). ${ }^{4}$ Both the estimated waning of zoster-vaccine efficacy ${ }^{5}$ and the relative high cost of zoster vaccine led to the fact that many countries worldwide remain reluctant to universally implement or reimburse the vaccine.

We believe that the immunogenicity equivalence of the dose-sparing, and thus cost-sparing, intradermal vaccine could have a major effect on zoster vaccine cost-effectiveness analyses. Of course, the clinical efficacy still needs to be addressed for this intradermal formulation, but the presented immunogenicity results are promising. It is also justifiable to argue that the intradermal deposition of varicella-zoster vaccine antigen might even induce a better longterm cellular immune response capable of preventing zoster with a higher vaccine efficacy than the subcutaneous zoster vaccine. Future cost-effectiveness analyses focused on intradermal zoster vaccine should thus differentiate between a lower-dosed and less expensive intradermal zoster vaccine and a similarly dosed intradermal zoster vaccine with higher clinical efficacy and lower waning rate.

Importantly, intradermal zoster vaccine might not only be promising to reduce zoster burden of illness, but also chickenpox related burden of illness. Hope-Simpson ${ }^{6}$ already hypothesised in 1965 that re-exposure to chickenpox could boost the immune response in varicella-zoster virus-experienced adults (so-called exogenous boosting hypothesis) and thereby reduce the risk of zoster. Consequently, many mathematical modelling analyses focused on universal chickenpox vaccination have predicted that the reduction of varicellazoster virus circulation caused by chickenpox vaccination could actually induce a 
(temporarily) increase of zoster occurrence. ${ }^{7-9}$ A recent systematic multidisciplinary review concluded that exogenous boosting existed, although the true magnitude still remains an issue for debate. ${ }^{10}$ Cost-effectiveness analyses were rather negative in regard of universal chickenpox vaccination because of the predicted increase of zoster incidence. These analyses concluded that subcutaneous zoster vaccine was unlikely to mitigate the predicted increase of zoster. ${ }^{7,11}$ Currently, only a few countries worldwide (including the USA, Australia, Taiwan, Canada, Germany, and Greece) have implemented universal chickenpox vaccination, whereas many more remain hesitant. The more efficacious intradermal zoster vaccine might thus not only reduce burden of illness in the elderly population, but could also even the path for universal childhood chickenpox vaccination and have an indirect positive effect on reducing chickenpox related burden of illness.

Finally, it is important to realise that all zoster vaccine-related cost-effectiveness analyses to date have been done with data from the Merck Zostavax trial. GlaxoSmithKline has recently presented more than $94 \%$ effectiveness in preventing zoster 3 years after vaccination with their varicella-zoster virus glycoprotein $E$ subunit vaccine. ${ }^{12}$ The GlaxoSmithKline subunit and Merck intradermal zoster vaccines promise an interesting future for herpes zoster vaccination.

\section{References}

${ }^{1}$ Beals CR, Railkar RA, Schaeff er AK, et al. Immune response and reactogenicity of intradermal administration versus subcutaneous administration of varicella-zoster virus vaccine: an exploratory, randomised, partly blinded trial. Lancet Infect Dis 2016; published online April 6. http://dx.doi.org/10.1016/S1473-3099(16)00133-X.

${ }^{2}$ Levin MJ, Oxman MN, Zhang JH, et al. Varicella-zoster virus-specific immune responses in elderly recipients of a herpes zoster vaccine. J Infect Dis 2008; 197: 825-35.

${ }^{3}$ Oxman MN, Levin MJ, Johnson GR, et al. A vaccine to prevent herpes zoster and postherpetic neuralgia in older adults. N Engl J Med 2005; 352: 2271-84.

${ }^{4}$ Morrison VA, Johnson GR, Schmader KE, et al. Long-term persistence of zoster vaccine efficacy. Clin Infect Dis 2015; 60: 900-09.

${ }^{5}$ Bilcke J, Ogunjimi B, Hulstaert F, Van Damme P, Hens N, Beutels P. Estimating the agespecifi $\mathrm{C}$ duration of herpes zoster vaccine protection: a matter of model choice? Vaccine 2012; 30: 2795-800.

${ }^{6}$ Hope-Simpson RE. The nature of herpes zoster: a long-term study and a new hypothesis. Proc R Soc Med 1965; 58: 9-20.

${ }^{7}$ Bilcke J, van Hoek AJ, Beutels P. Childhood varicella-zoster virus vaccination in Belgium: cost-eff ective only in the long run or without exogenous boosting? Hum Vaccin Immunother 2013; 9: 812-22.

${ }^{8}$ Brisson M, Edmunds WJ, Gay NJ, Law B, De Serres G. Modelling the impact of immunization on the epidemiology of varicella zoster virus. Epidemiol Infect 2000; 125: 65169.

${ }^{9}$ Ogunjimi B, Willem L, Beutels $\mathrm{P}$, Hens N. Integrating between-host transmission and withinhost immunity to analyze the impact of varicella vaccination on zoster. Elife 2015; 4: e07116.

${ }^{10}$ Ogunjimi B, Van Damme P, Beutels P. Herpes zoster risk reduction through exposure to chickenpox patients: a systematic multidisciplinary review. PLoS One 2013; 8: e66485.

${ }_{11}$ van Hoek AJ, Melegaro A, Gay N, Bilcke J, Edmunds WJ. The cost-effectiveness of varicella and combined varicella and herpes zoster vaccination programmes in the United Kingdom. Vaccine 2012; 30: 1225-34.

${ }^{12}$ Lal H, Cunningham AL, Godeaux O, et al. Efficacy of an adjuvanted herpes zoster subunit vaccine in older adults. N Engl J Med 2015; 372: 2087-96. 